Pacific Journal of Mathematic 


\title{
COMMUTATIVE SEMIGROUPS WHICH ARE ALMOST FINITE
}

\author{
Bruce A. Jensen and Donald W. Miller
}

\begin{abstract}
Semigroups satisfying certain finiteness conditions are studied. It is shown that an infinite semigroup $S$ every proper subsemigroup of which is finite is a group; thus in particular if $S$ is commutative then it is isomorphic to the group $Z\left(p^{\infty}\right)$ for some prime $p$. An infinite commutative semigroup every proper homomorph of which is finite is shown to be imbeddable in an infinite cyclic group with zero element adjoined and its structure is described.
\end{abstract}

In his monograph on infinite abelian groups Kaplansky [2] includes the following two exercises concerning an infinite abelian group $G$ :

(I) If every proper subgroup of $G$ is finite then, for some prime $p, G$ is isomorphic to $Z\left(p^{\infty}\right)$.

(II) If every proper homomorph of $G$ is finite then $G$ is an infinite cyclic group.

The converse of each of these implications is, of course, also true.

It is natural to ask what conclusions can be drawn for commutative semigroups under analogous hypotheses. In $\S 1$ it is shown that if $S$ is an infinite semigroup each of whose proper subsemigroups is finite then $S$ is a group. Thus in particular if $S$ is commutative the conclusion of (II) is obtained.

A semigroup $S$ is said to be homomorphically finite, or, for brevity, $H F$, if $S$ is infinite while each proper homomorph of $S$ is finite. Section 2 is devoted to showing that an infinite commutative semigroup $S$ is $H F$ if and only if $S$ is imbeddable in an infinite cyclic group. A description of all such semigroups is given.

The notation and terminology used in this paper follow that of Clifford and Preston [1].

1. Infinite semigroups whose proper subsemigroups are finite. We begin with a lemma.

Lemma 1. Let $S$ be an infinite semigroup having no proper infinite subsemigroups. Then:

(i) $S$ is periodic;

(ii) $S^{2}=S$;

(iii) $S$ is not nil.

Proof. (i) If $a \in S$ then either $\langle a\rangle$, the subsemigroup of $S$ 
generated by $a$, is finite or $S=\langle a\rangle$ is cyclic. In the latter case, however, $S$ contains the proper infinite subsemigroup $\left\langle a^{2}\right\rangle$, contrary to hypothesis.

(ii) If $x \in S \backslash S^{2}$, the complement of $S^{2}$ in $S$, then $S \backslash\{x\}$ is a proper infinite subsemigroup of $S$. Hence $S=S^{2}$.

(iii) If $S$ contains no zero element, (iii) holds by default. Hence suppose that 0 is a zero element of $S$ and that $S$ is nil. By (ii) we can choose $a \in S$ such that $a S \neq 0$. Hence either $a S$ is finite or $a S=S$. If $a S=S$ then $S=a^{n} S$ for every positive integer $n$ so, since $S$ is nil, $S=a^{k} S=0 S=0$ for some positive integer $k$, a contradiction. Hence assume that $a S=\left\{x_{0}, x_{1}, \cdots, x_{n}\right\}$, where $n>0, x_{0}=0$ and $x_{i} \neq x_{j}$ for $i \neq j$. For $i=0,1, \cdots, n$, define

$$
S_{i}=\left\{y \in S \mid a y=x_{i}\right\}
$$

and define a binary relation $\leqq$ on the set $\mathscr{S}$ of all $S_{i}$ by stipulating that $S_{i} \leqq S_{j}$ if and only if there exists $s$ in $S^{1}$ such that $x_{j}=x_{i} s$. Clearly $\leqq$ is reflexive and transitive on $\mathscr{S}$. Moreover suppose $S_{i} \leqq S_{j}$ and $S_{j} \leqq S_{i}$, say $x_{j}=x_{i} s$ and $x_{i}=x_{j} t$, where $s, t \in S^{1}$. Then

$$
x_{j}=x_{j}(t s)^{k}, \quad k=1,2,3, \cdots \text {. }
$$

Since $S$ is nil this implies that either $x_{j}=0$, whence also $x_{i}=0$, or $t s \notin S$. In the latter case, $s=t=1$ so again $x_{i}=x_{j}$. Thus $\leqq$ is a partial ordering of $\mathscr{S}$.

Evidently $S_{i} \leqq S_{0}$ for $i=0,1, \cdots, n$. Moreover there must exist an integer $N, 1 \leqq N \leqq n$, such that

$$
S_{i} \leqq S_{N} \text { implies } i=N \text {, all } S_{i} \in \mathscr{S} \text {. }
$$

Let $y \in S_{x}$. By (ii) $y=u v$ for some $u, v \in S$. Since $\mathscr{S}$ describes a partition of $S, u \in S_{i}$ for exactly one $i, 0 \leqq i \leqq n$. Therefore $x_{N}=$ $a y=a u v=x_{i} v$ so $S_{i} \leqq S_{N}$ whence, by (1), $i=N$ and $x_{N}=x_{N} v$. Consequently $x_{N}=x_{N} v^{k}$ for $k=1,2,3, \cdots$ so $x_{N}=0=x_{0}$, contrary to $N>0$. This establishes (iii).

THEOREM 1. If $S$ is an infinite semigroup each of whose proper subsemigroups is finite then $S$ is a group.

Proof. Let $A_{1}=\{x \in S \mid x S$ is finite $\}$ and $A_{2}=\{x \in S \mid x S=S\}$. For $i=1,2$, if $A_{i} \neq \varnothing$ (the null set) then $A_{i}$ is a subsemigroup of $S$. Thus, since $A_{1}$ and $A_{2}$ partition $S$, either $A_{1}=S$ and $A_{2}=\varnothing$ or vice versa. An analogous argument on the principal left ideals of $S$ leads to the conclusion that $S$ satisfies exactly one of the following:

(i) $x S$ and $S x$ are finite, all $x \in S$;

(ii) $x S$ is finite and $S x=S$, all $x \in S$; 
(iii) $x S=S$ and $S x$ is finite, all $x \in S$;

(iv) $x S=S x=S$, all $x \in S$.

Denote the set of idempotents of $S$ by $E$; since $S$ is periodic, $E \neq \varnothing$.

Case (i). If $E$ is finite then $S^{1} E S^{1}$ is a finite ideal of $S$ and the Rees factor semigroup $S / S^{1} E S^{1}$ is an infinite semigroup having only finite proper subsemigroups. However some power of each element of $S$ is idempotent so $S / S^{1} E S^{1}$ is nil. Since this contradicts Lemma 1, $E$ must be infinite.

For each $e \in E$ define the subsemigroups $L_{e}$ and $R_{e}$ of $S$ by

$$
L_{e}=\{x \in S \mid x e=e\}, \quad R_{e}=\{y \in S \mid e y=e\} .
$$

If $L_{e}=L_{f}=R_{e}=R_{f}=S$ for some $e, f \in E$ then $e=e f=f$. Hence, since $E$ is infinite, there must exist an $e$ in $E$ such that either $L_{e} \neq S$ or $R_{e} \neq S$. Assume the former; then $L_{e}$ is finite. Therefore $E^{\prime}=E \backslash L_{e}$ is an infinite subset of $S$ so $E^{\prime}$ generates $S$. Consequently there exist elements $f_{1}, f_{2}, \cdots, f_{k}$ of $E^{\prime}$ such that $f_{1} f_{2} \cdots f_{k}=e$. Thus $f_{1} e=e$ so $f_{1} \in L_{e}$, contradicting $f_{1} \in E^{\prime}$. The assumption that $R_{e} \neq S$ leads to a similar contradiction, so case (i) is eliminated.

Cases (ii) and (iii) are left-right duals so only one of them need be considered. Suppose then that for each $x$ in $S, S x$ is finite and $x S=S$. Then $e x=x$ for all $e \in E$ so $E$ is a right zero subsemigroup of $S$. Thus if $e \in E$ and $E^{\prime}=E \backslash\{e\}$ then $E^{\prime}$ is either empty or a pro per subsemigroup of $S$. In either case we conclude that $E$ is finite.

Suppose $E=\{e\}$. Then, since $S$ is periodic, there corresponds to each element $x$ of $S$ a positive integer $n=n(x)$ such that $x^{n}=e$. Therefore $x e=x^{n+1}=e x=x$ so $S e=S$, contradicting the finiteness of $S e$. Hence $E$ has order $k>1$, say $E=\left\{e_{1}, \cdots, e_{k}\right\}$. For each $i$, $1 \leqq i \leqq k$, define $T_{i}=\left\{x \in S \mid e_{i} \in\langle x\rangle\right\}$. By the periodicity of $S$, the set $\left\{T_{1}, \cdots, T_{k}\right\}$ is a partition of $S$. If $x \in T_{i}$, say $x^{m}=e_{i}$, then, as above, it follows that $x e_{i}=x$, so that $T_{i} \subseteq U_{i}=\left\{x \in S \mid x e_{i}=x\right\}$, $i=1, \cdots, k$. Conversely let $x \in U_{i}$ and suppose $x^{n}=e_{j}$ for some $n>0$ and $j, 1 \leqq j \leqq k$. As above,

$$
e_{j}=x^{n}=x^{n} e_{i}=e_{j} e_{i}=e_{i} .
$$

Thus $i=j$ so $U_{i} \leqq T_{i}$. Therefore $T_{i}=U_{i}$ is a subsemigroup of $S$ for $i=1, \cdots, k$. But since $S=\bigcup_{1}^{k} T_{i}$ and $k>1$ it follows that at least one of the $T_{i}$ is an infinite proper subsemigroup of $S$, a contradiction.

This leaves only case (iv). Thus $S$ is a group.

Combining Theorem 1 with Kaplansky's Exercise (I) we then have the following result.

THEOREM 2. If $S$ is an infinite commutative semigroup each of 
whose proper subsemigroups is finite then $S$ is isomorphic to the group $Z\left(p^{\infty}\right)$ for some prime $p$.

2. Commutative $H F$ semigroups. An $H F$ (or homomorphically finite) semigroup is defined to be an infinite semigroup each of whose noninjective homomorphisms has finite image. An $H F$ group is an $H F$ semigroup which is also a group, e.g., the infinite cyclic group or any infinite simple group. The following two results are immediate consequences of these definitions.

LEMma 2. Every proper nonzero ideal of an $H F$ semigroup $S$ has finite complement in $S$.

LEMmA 3. If $S$ is an infinite semigroup then either all or none of $S, S^{1}, S^{0}$ and $\left(S^{1}\right)^{0}$ are $H F$ semigroups.

L. Rédei [3, Satz 82] has given essentially the following characterization of the subsemigroups of the additive semigroup $N$ of positive integers.

Lemma 4. (Rédei). Let $N$ be the additive semigroup of all positive integers and let $d, r \in N$. Define

$$
I=\{n d \mid n \in N, n d \geqq r\}
$$

and let $A$ be any subset of $d N \backslash I$ such that $A+A \subseteq A \cup I$. Then $S=S(d, r, A)$ is a subsemigroup of $N$, and every subsemigroup of $N$ is so obtainable. Furthermore, for suitable choice of $r^{\prime}$ and $A^{\prime}, S(d, r, A) \cong S\left(1, r^{\prime}, A^{\prime}\right)$.

Rédei's result can easily be extended to the additive group $Z$ of all integers.

LEMMA 5. If $S$ is a nonzero subsemigroup of $Z$ then either $S$ is isomorphic to $Z$ or, for suitable $r \in N$ and $A \subseteq r N, S$ is isomorphic to $S(1, r, A)$ with or without an adjoined identity.

Proof. In view of the isomorphism between the subsemigroups $N$ and $-N=(-1) N$ of $Z$ we need only consider those subsemigroups of $Z$ which contain both a positive and a negative integer. Let $S$ be such a semigroup and let $S_{1}=S \cap N, S_{2}=S \cap(-N)$. For $i=1,2$ it follows from Lemma 4 that $S_{i}$ is isomorphic to $S\left(d_{i}, r_{\imath}, A_{i}\right)$ for suitable $d_{i}, r_{i}, \in N$ and $A_{i} \subseteq r_{i} N \backslash I_{i}$, where $I_{i}=\left\{n d_{i} \mid n \in N, n d_{i} \geqq r_{i}\right\}$. Moreover $u d_{1} \in S_{1}$ and $-v d_{2} \in S_{2}$, and hence $u d_{1}-v d_{2} \in S$, for all sufficiently large integers $u$ and $v$. Therefore $\left(d_{1}, d_{2}\right) \in S$ so $d_{1}=d_{2}$. It then follows that $S$ is the cyclic subgroup of $Z$ generated by $d$. 
Commutative $H F$ semigroups can now be characterized.

THEOREM 3. Let $S$ be an infinite commutative semigroup. Then $S$ is homomorphically finite if and only if $S$ is imbeddable in an infinite cyclic group with adjoined zero. If this is the case then either $S$ is itself an infinite cyclic group or $S$ is isomorphic to a subsemigroup $\bar{S}$ of the additive semigroup of all nonnegative integers with zero element $\infty$ adjoined. In the latter event there exist positive integers $a_{1}, \cdots, a_{k}$ and $r$ such that

$$
\bar{S}=\left\{a_{1}, a_{2}, \cdots, a_{k}\right\} \cup\{n \mid n \geqq r\},
$$

possibly with adjoined zero element $\infty$.

Proof. Let $S$ be a subsemigroup of the additive group of integers with adjoined zero $\infty$. By Lemma 3 there is no loss of generality in assuming that $\infty \notin S$. Hence by Lemma 5 either $S$ is an infinite cyclic group, and thus is homomorphically finite, or $S$ is isomorphic to some semigroup $S(1, r, A)$, so that $S=I \cup A$, where $I=\{n \mid n \in N, n \geqq r)$ is an ideal of $S$. Assuming the latter, let $\sigma$ be a nontrivial congruence on $S$ which is not one-to-one, so that $a \sigma b$ for two distinct elements $a, b$ of $S$. Then $(n a) \sigma(n b)$ for all $n>0$ so $\sigma$ is not one-to-one on $I$, whence we can assume that $a, b \in I$, with $a<b$. Then $(a+(r+k))$ $\sigma(b+(r+k))$ for each $k \geqq 0$.

Define $m=b-a$ and let $x, y \in I$, with $x, y \geqq a+r$ and $x \equiv y(\bmod m)$, say $x=a+s, y=a+s+t m$, where $s, t \in N$ and $s \geqq r$. Since $a \sigma b$ then $(a+s) \sigma(b+s)$, i.e., $(a+s) \sigma(a+s+m)$. Thus by induction $(a+s) \sigma(a+s+t m)$ so $x \sigma y$. It follows that the factor semigroup $I / \sigma$ is finite so by the finiteness of $A, S / \sigma$ is also finite. Therefore $S$ is an $H F$-semigroup.

Conversely let $S$ be a commutative $H F$ semigroup. For each $c$ in $S$ define the congruence $\sigma_{c}$ on $S$ by

$$
a \sigma_{c} b \text { if and only if } a c=b c \text {, all } a, b \in S .
$$

If there exists an element $c$ in $S$ such that $S / \sigma_{c}$ is finite then the ideal $S c$ of $S$ would also be finite, which, in the light of Lemma 2, would contradict the assumption that $S$ is infinite unless $S c=0$.

Suppose $S c=0$ and let $J=\{x \in S \mid S x=0\}$. Then $J$ is an ideal of $S$ so either $J=0$ or $S / J$ is finite. In the latter case, we conclude that $S^{2}$ is also finite; thus $S^{2}=0$ since $S^{2}$ and $S / S^{2}$ cannot both be finite. However it is evident that the condition $S^{2}=0$ cannot hold in an $H F$ semigroup $S$, so $J=0$. Hence $S c=0$ only if $c=0$.

Thus $S / \sigma_{c}$ is infinite, and $\sigma_{c}$ is one-to-one, for all $c$ in $S \backslash 0$ so $S$ is a commutative cancellative semigroup, possibly with an adjoined zero. In any event, $S$ contains no proper zero divisors. 
Let $T=S \backslash 0$ or $T=S$ according as $S$ does or does not contain a zero element. Let $G$ denote the group of quotients of $T$ and regard $T$ as a subsemigroup of $G$, in the usual manner. Suppose $\sigma$ is a congruence on $G$ which is not one-to-one, say $(a / b) \sigma(c / d)$, where $a$, $b, c, d \in S$ and $a d \neq b c$. Then $((a / b) b d) \sigma((c / d) b d)$, i.e., $(a d) \sigma(b c)$. Consequently $\sigma^{\prime}$, the restriction of $\sigma$ to $S$, is not one-to-one on $T$ so $T / \sigma^{\prime}$ is finite.

For $x \in T$ let $[x]$ and $[x]^{\prime}$ denote the $\sigma$-class of $G$ and the $\sigma^{\prime}$-class of $T$, respectively, containing $x$. Then the homomorphism of $T / \sigma^{\prime}$ into $G / \sigma$ defined by $[x]^{\prime} \rightarrow[x]$, all $x \in T$, is injective. Thus $T / \sigma^{\prime}$ is cancellative and hence is a finite abelian group. It is readily verified that the mapping of $S / \sigma^{\prime}$ defined by $[x] \rightarrow[x]^{\prime}$, all $x \in S$, is an isomorphism of $S / \sigma^{\prime}$ onto $G / \sigma$. Therefore $G / \sigma$ is also finite so $G$ is an abelian $H F$-group. Thus by Kaplansky's Exercise II, $G$ is cyclic.

An application of Lemma 5 now completes the proof.

\section{REFERENCES}

1. A. H. Clifford and G. B. Preston, The Algebraic Theory of Semigroups, Vol. 1, American Mathematical Society, Providence, Rhode Island, 1961.

2. I. Kaplansky, Infinite Abelian Groups, The University of Michigan Press, Ann Arbor, Michigan, 1954.

3. L. Rédei, Theorie der Endlich Erzeugbaren Kommutativen Halbgruppen, B. G. Teubner Verlagsgesellschaft Leipzig, 1963.

Received October 31, 1967. Under the partial support of a N.S.F. Nebraska Cooperative Fellowship. 


\section{PACIFIC JOURNAL OF MATHEMATICS}

\section{EDITORS}

\section{H. ROYDEN}

Stanford University

Stanford, California

\section{J. Dugundu}

Department of Mathematics University of Southern California Los Angeles, California 90007

RICHARD ARENS

University of California Los Angeles, California 90024

ASSOCIATE EDITORS
E. F. Beckenbach
B. H. NEUMANN
F. WoLF
K. YosidA

\section{SUPPORTING INSTITUTIONS}

\author{
UNIVERSITY OF BRITISH COLUMBIA \\ CALIFORNIA INSTITUTE OF TECHNOLOGY \\ UNIVERSITY OF CALIFORNIA \\ MONTANA STATE UNIVERSITY \\ UNIVERSITY OF NEVADA \\ NEW MEXICO STATE UNIVERSITY \\ OREGON STATE UNIVERSITY \\ UNIVERSITY OF OREGON \\ OSAKA UNIVERSITY \\ UNIVERSITY OF SOUTHERN CALIFORNIA
}

STANFORD UNIVERSITY

UNIVERSITY OF TOKYO

UNIVERSITY OF UTAH

WASHINGTON STATE UNIVERSITY

UNIVERSITY OF WASHINGTON

AMERICAN MATHEMATICAL SOCIETY
CHEVRON RESEARCH CORPORATION
TRW SYSTEMS

AMERICAN MATHEMATICAL SOCIETY

TRW SYSTEMS

NAVAL WEAPONS CENTER

Mathematical papers intended for publication in the Pacific Journal of Mathematics should be in typed form or offset-reproduced, double spaced with large margins. Underline Greek letters in red, German in green, and script in blue. The first paragraph or two must be capable of being used separately as a synopsis of the entire paper. It should not contain references to the bibliography. Manuscripts, in duplicate if possible, may be sent to any one of the four editors. All other communications to the editors should be addressed to the managing editor, Richard Arens, University of California, Los Angeles, California 90024.

Each author of each article receives 50 reprints free of charge; additional copies may be obtained at cost in multiples of 50 .

The Pacific Journal of Mathematics is published monthly. Effective with Volume 16 the price per volume (3 numbers) is $\$ 8.00$; single issues, $\$ 3.00$. Special price for current issues to individual faculty members of supporting institutions and to individual members of the American Mathematical Society: $\$ 4.00$ per volume; single issues $\$ 1.50$. Back numbers are available.

Subscriptions, orders for back numbers, and changes of address should be sent to Pacific Journal of Mathematics, 103 Highland Boulevard, Berkeley 8, California.

Printed at Kokusai Bunken Insatsusha (International Academic Printing Co., Ltd.), 7-17, Fujimi 2-chome, Chiyoda-ku, Tokyo, Japan.

PUBLISHED BY PACIFIC JOURNAL OF MATHEMATICS, A NON-PROFIT CORPORATION

The Supporting Institutions listed above contribute to the cost of publication of this Journal, but they are not owners of publishers and have no responsibility for its content or policies. 


\section{Pacific Journal of Mathematics \\ Vol. 27, No. 3}

March, 1968

Charles A. Akemann, Invariant subspaces of $C(G) \ldots \ldots \ldots \ldots \ldots \ldots . \ldots 41$

Dan Amir and Zvi Ziegler, Generalized convexity cones and their duals ... . 425

Raymond Balbes, On ( $J, M, \mathrm{~m})$-extensions of order sums of distributive

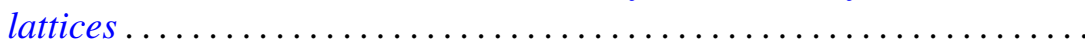

Jan-Erik Björk, Extensions of the maximal ideal space of a function algebra ........................................ 453

Frank Castagna, Sums of automorphisms of a primary abelian group ...... 463

Theodore Seio Chihara, On determinate Hamburger moment problems ..... .

Zeev Ditzian, Convolution transforms whose inversion function has complex

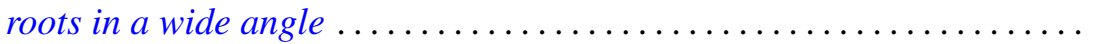

Myron Goldstein, On a paper of Rao .

Velmer B. Headley and Charles Andrew Swanson, Oscillation criteria for

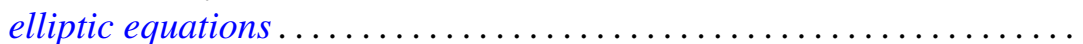

John Willard Heidel, Qualitative behavior of solutions of a third order nonlinear differential equation............................

Alan Carleton Hindmarsh, Pick's conditions and analyticity.............

Bruce Ansgar Jensen and Donald Wright Miller, Commutative semigroups

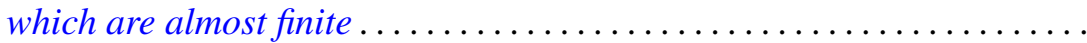

Lynn Clifford Kurtz and Don Harrell Tucker, An extended form of the

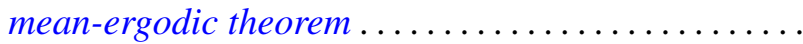

S. P. Lloyd, Feller boundary induced by a transition operator ...

Henry B. Mann, Josephine Mitchell and Lowell Schoenfeld, A new proof of the maximum principle for doubly-harmonic functions ...

Robert Einsohn Mosher, The product formula for the third obstruction ..

Sam Bernard Nadler, Jr., Sequences of contractions and fixed points ...

Eric Albert Nordgren, Invariant subspaces of a direct sum of weighted shifts...

Fred Richman, Thin abelian p-groups ...

Jordan Tobias Rosenbaum, Simultaneous interpolation in $\mathrm{H}_{2}$. II ...

Charles Thomas Scarborough, Minimal Urysohn spaces .

Malcolm Jay Sherman, Disjoint invariant subspaces..... .

Joel John Westman, Harmonic analysis on groupoids....

621

William Jennings Wickless, Quasi-isomorphism and TFM

Minoru Hasegawa, Correction to "On the convergence of resolvents of operators" 